valve endocarditis caused by Acinetobacter calcoaceticus subsp. Iwoffii. J Clin Microbiol 1987;25:955-7.

9. Fuchs GJ III, Jaffe N, Pickering LK. Acinetobacter calcoaceticus sepsis in children with malignancies. Pediatr Infect Dis 1986;5:545-9.

10. Seifert H, Strate A, Schulze A, Pulverer G. Vascular catheter-related

\section{Myelitis Due to Varicella-Zoster Virus in an Immunocompromised Patient Without a Cutaneous Rash}

SIR-Immunosuppression increases the susceptibility of patients to varicella-zoster virus (VZV) reactivation and to severe visceral dissemination, particularly in the CNS. It is easy to make the diagnosis of $\mathrm{VZV}$ reactivation when the patient presents with a typical vesicular rash. However, VZV reactivation can occur without rash (zoster sine herpete) [1], and such cases are much more difficult to diagnose, as is exemplified by our own case.

A 35-year-old iv drug addict initially presented to the hospital in 1989 with Pneumocystis carinii pneumonia, oral thrush, and oral herpes ulcers. He was also infected with the human immunodeficiency virus (HIV). Over the next 3 years, he received therapy with oral zidovudine, aerosolized pentamidine, and oral fluconazole. The patient's immunosuppression progressed; 1 month before his second admission the CD4 cell count decreased to $32 / \mathrm{mm}^{3}$, the $\beta_{2}$-microglobulin level rose to $6.5 \mathrm{mg} / \mathrm{L}$, and the p24 antigen level rose to $94 \mathrm{pg} / \mathrm{mL}$. Three weeks before his second admission he had a fever (temperature to $38.5^{\circ} \mathrm{C}$ ), headaches, and increased frequency of urination. Several days later, oliguria and bilateral leg weakness were noted; at the time of admission the weakness had progressed to paraplegia, and the patient also had urinary incontinence and intermittent diplopia.

Physical examination showed an afebrile, cachectic, dehydrated patient with diminished mentation. No cutaneous lesions were observed. The patient had mild neck stiffness and no abdominal skin reflexes; paraplegia was still noted, and there was decreased sensitivity below the T-8 level. A bladder catheter yielded $1.5 \mathrm{~L}$ of urine.

A complete blood cell count revealed only macrocytosis and mild thrombocytopenia. A blood chemistry screening panel revealed mild azotemia and rhabdomyolysis. The level of glucose in CSF was $3.7 \mathrm{mmol} / \mathrm{L}$, and the level of protein in CSF was 684 $\mathrm{mg} / \mathrm{dL}$. The white blood cell count in CSF was $32 / \mathrm{mm}^{3}(50 \%$ lymphocytes, $32 \%$ reticulohistiocytes, $10 \%$ neutrophils, and $8 \%$ plasmocytes), and there were no red blood cells. Findings of a gram stain of CSF and the results of a cryptococcal antigen test were negative. Cultures of blood and urine were negative for bacteria, mycobacteria, and viruses, and culture of urine yielded cytomegalovirus (CMV). CSF samples were inoculated on hu-

Reprints or correspondence: Dr. Pascal R. A. Meylan, Institut de Microbiologie. Centre Hospitalier Universitaire Vaudois, $\mathrm{CH}-1011$ Lausanne, Switzerland.

Clinical Infectious Diseases 1995;20:206-8

(C) 1995 by The University of Chicago. All rights reserved. 1058-4838/95/2001-0042\$02.00 bloodstream infection due to Acinetobacter johnsonii (formerly Acinetobacter calcoaceticus var. Iwoffii): report of 13 cases. Clin Infect Dis 1993; 17:632-6.

11. Kaplan MS, Wechsler M. Benson MC. Urologic manifestations of AIDS. Urology 1987;30:441-3

man embryonic lung fibroblasts and culture of these samples did not yield any virus.

Serum drawn at the time of admission was positive by CF at a dilution of 1:16 for herpes simplex virus (HSV) and for VZV (antigens were obtained from Virion [Zurich]). Specific IgG was determined to be present in serum by ELISA (Clark Laboratories, Jamestown, NY). Index values (optical density ratio of test sample over cutoff value for positivity [ $\geqslant 1.0]$ ) of $\operatorname{lgG}$ were 3.5 for HSV, 3.8 for VZV, and 2.1 for CMV. No specific IgM to any of the three viruses was detected by ELISA (Sorin Biomedica, Saluggia, Italy) or by immunofluorescence assay (Gull Laboratories, Salt Lake City). The results of serological studies were negative for syphilis. A computed tomogram showed only cerebral atrophy.

The spinal cord was examined by magnetic resonance imaging (MRI) at 1.5 Tesla. On T l-weighted images, the spinal cord was heterogeneous, and there were multiple focal decreases in signal intensity. The T2-weighted images showed multiple focal lesions of the spinal cord with a strong increase in signal intensity; the maximum density of lesions was at the level of the T-9 to $\mathrm{T}-10$ vertebrae (figure 1). After contrast injection, the sagittal Tl-weighted image showed abnormal foci of increased signal in the spinal cord that were much smaller than areas of increased $\mathrm{T} 2$ signal. This finding was suggestive of a multifocal inflammatory process, with enhancement of the contrast material surrounding small areas of necrosis.

The patient died suddenly 6 days after admission. At postmortem examination the cerebral and cerebellar hemispheres were grossly normal, and only a few leukocytes and macrophages had infiltrated the leptomeninges. Greyish discoloration of a subependymal zone at the level of the brain stem was consistent with hemorrhagic necrosis and some leukocytic infiltration. Several intranuclear inclusions were seen, mostly in the ependymal cells. Examination of the spinal cord revealed thickened, brownish leptomeninges, and tumefaction with softening at the T-8 to T- 10 level.

Microscopic examination revealed hemorrhagic necrosis with marked leukocytic infiltration along the spinal cord and radicular nerves, with complete destruction of the spinal cord parenchyma at the T8-10 levels. There was leukocytic infiltration of the walls of several leptomeningeal and intraparenchymatous vessels, and this infiltration was often associated with necrosis and secondary thrombosis. Ganglional cell loss and (in some of the remaining cells) Cowdry type A intranuclear inclusions were observed in a spinal ganglion at the T- 10 level, but no inflammatory reaction or hemorrhagic necrosis was noted.

Histologic examination did not reveal HIV encephalopathy or vacuolar myelopathy. CSF samples harvested at postmortem examination were inoculated on human embryonic lung fibroblasts for viral cultures. Culture of a specimen of the frontal lobe of the brain admixed with CSF yielded CMV, while culture of a 


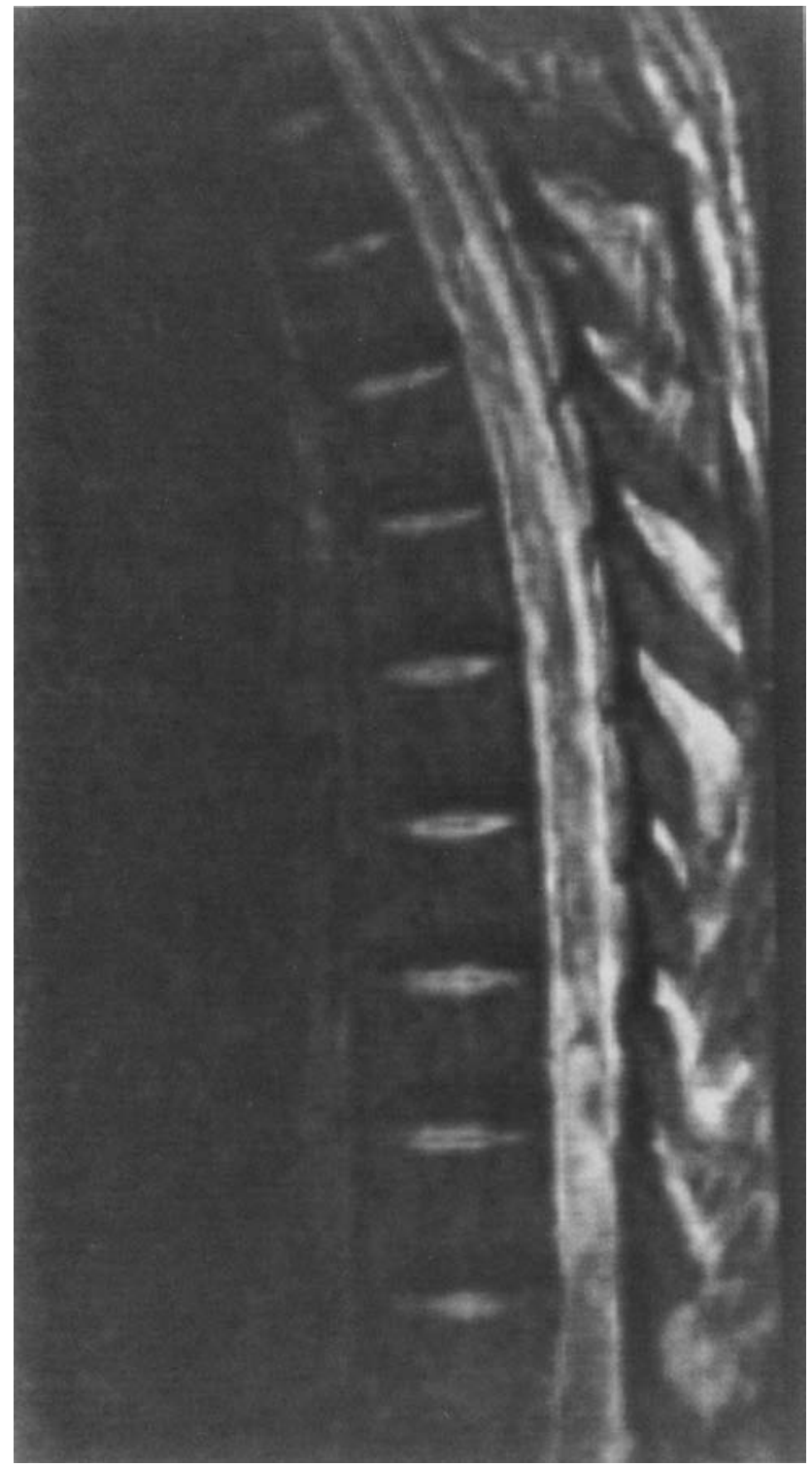

Figure 1. MRI scan of the dorsal spinal cord of an HIV-infected patient who had VZV myelitis without cutaneous rash. The T2-weighted image of the midsagittal plane (TR 2500, TE 90) shows multiple foci of increased signal intensity with a maximum density of lesions at the T8-10 level.

CSF specimen taken from the T-9 level of the spinal cord yielded VZV. This virus was characterized by indirect immunofluorescence with use of monoclonal antibodies (IU1 antibody was used for VZV, E-13 antibody for CMV; Biosoft [Clonatec. Paris]).

The case described herein demonstrates that VZV reactivation and dissemination to the CNS can occur in the absence of cutaneous lesions. The occurrence of herpes zoster symptoms in the absence of a typical rash, preceding the rash, or following the rash is termed zoster sine herpete $[1,2]$. If the course of zoster sine herpete is uncomplicated, a tentative diagnosis can be made on the basis of the pattern of radicular pain; this diagnosis can be supported in some cases by the eventual appearance of a rash from several days to months after pain appeared [1]. A signifi- cant rise in antibodies to VZV was observed in only one such case, and no case of uncomplicated zoster sine herpete has ever been virologically documented.

In contrast, the occurrence of VZV reactivation without rash has been demonstrated by several cases of reactivation associated with visceral dissemination; in these cases, VZV infection was documented by various methods. Mayo and Booss [3] reported the cases of 10 patients with neurological disease and serological evidence for VZV reactivation. Heller et al. [4] observed a case of transverse myelitis in an otherwise healthy patient; in this case, VZV was cultured from CSF specimens. In addition, this patient did not develop a response to VZV antibody. Finally, a patient with $\mathrm{T}$ cell lymphoma developed fatal meningoradiculitis without rash. At death, hemorrhagic necrosis and Cowdry type A inclusion bodies were seen in the meninges. nerve roots, viscera, and lymph nodes. VZV antigen and nucleic acid were demonstrated by immunohistochemical studies and by in situ hybridization in the same tissues [1].

To our knowledge, we report the first case of VZV myelitis without cutaneous manifestations in a patient with AIDS. This is also the first time that there was complete pathological and microbiological documentation of the necrotic myelitis, as culture of a spinal cord specimen yielded VZV. VZV myelitis was not considered in the differential diagnosis before the postmortem examination was performed because the patient did not have a rash or radicular pain suggestive of this disease and because culture of CSF specimens did not yield VZV. After compression of the spinal cord was ruled out on the basis of findings on an MRI scan, two diagnoses were entertained. The first possible diagnosis was vacuolar myelopathy caused by HIV. However, vacuolar myelopathy is characterized mainly by spastic paraparesis, and a sensory level is distinctly unusual in this disease. Second, CMV radiculomyelitis is a cause of ascending paresis with hyporeflexia and is associated with characteristic neutrophilic pleocytosis and meningeal enhancement at the level of the spinal nerve roots $[5,6]$.

The ability of VZV to cause CNS disease, including encephalitis, myelitis, and cerebral vascular occlusion, is well established. Devinsky et al. [7] described 13 cases of herpes zoster myelitis and reviewed 33 cases from the literature. All cases occurred in association with clinical herpes zoster. Herpes zoster myelitis occurred more frequently among immunocompromised patients than among immunocompetent patients. Of note, myelitis preceded the rash by 8 days in one case. The clinical presentations of 38 patients included lower limb paresis (74\%) and sensory abnormalities ( $53 \%$ ), and $34 \%$ of the patients had a sensory level. Sphincter disturbances were noted in $61 \%$ of the cases. Thus. except for the terminal oculomotor symptoms, our patient's clinical signs and symptoms are consistent with VZV myelitis. The pathological and microbiological evidence in our case were consistent with CMV ventriculoencephalitis, a recently described syndrome with frequent cranial nerve deficits [8]; these nerve deficits may explain the terminal diplopia in patients with this syndrome.

Laboratory results were not diagnostic in our patient's case. IgM to VZV was not detected, and culture of CSF specimens from the initial spinal tap did not yield the virus. Indeed. VZV is only infrequently cultured from CSF specimens from patients with VZV infection of the CNS [4, 7]. Findings on an MRI scan 
were consistent with a multifocal inflammatory process, and there was enhancement of the contrast material surrounding small areas of necrosis. This process was disseminated throughout the spinal cord; the fact that the maximum number of lesions was at the T9-10 level was consistent with the clinical sensory level and the autopsy findings, perhaps reflecting the original level of $\mathrm{VZV}$ reactivation and entry into the spinal cord. In a recent report, an MRI study of a patient with VZV myelitis following herpes zoster involving the C3-4 dermatomes demonstrated an increased T2-weighted signal throughout the long segments of the spinal cord; the study revealed that the maximum number of lesions were in the cervical region, a finding consistent with those at autopsy [9]. In a retrospective review of this case, the sensory level in correlation with findings on the MRI scan might have been used to suggest the correct diagnosis.

The case described herein exemplifies the difficulty in establishing the cause of illness while patients who have viral infections of the CNS are alive. The polymerase chain reaction has been used to demonstrate VZV DNA in the CSF of patients with neurological complications associated with chickenpox or herpes zoster $[10,11]$. With use of the polymerase chain reaction, we will probably be able to diagnose VZV infections while patients are alive and thus initiate early antiviral treatment; such treatment will be beneficial as there is evidence that the progression of CNS deficits associated with VZV myelitis can be halted with use of appropriate antiviral agents, particularly acyclovir [7].

Pascal R. A. Meylan, Judith Miklossy, Anne Iten, Christiane Petignat, Reto Meuli, Jade Zufferey, and Roland Sahli

Division of Infectious Diseases, Departments of Radiology and Microbiology, Centre Hospitalier Universitaire Vaudois, Lausanne; and Division of Neuropatholog!, Universit!' Institute of Pathology, Lausanne. Suitzerland

\section{References}

1. Gilden DH. Dueland AN, Devlin ME, Mahalingam R, Cohrs R. Varicella-zoster virus reactivation without rash. J Infect Dis 1992; 166(suppl 1):S30-4.

2. Lewis GW. Zoster sine herpete. Br Med J 1958;2:418-21.

3. Mayo DR, Booss J. Varicella zoster-associated neurologic disease without skin lesions. Arch Neurol 1989;46:313-5.

4. Heller HM, Carnevale NT, Steigbigel RT. Varicella zoster virus transverse myelitis without cutaneous rash. Am J Med 1990;88:550-1.

5. de Gans J, Tiessens G, Portegies P, Tutuarima JA, Troost D. Predominance of polymorphonuclear leukocytes in cerebrospinal fluid of AIDS patients with cytomegalovirus polyradiculomyelitis. J Acquir Immune Defic Syndr 1990; 3:1 155-8

6. Talpos D. Tien RD. Hesselink JR. Magnetic resonance imaging of AIDS-related polyradiculopathy. Neurology 1991;41:1995-7.

7. Devinsky O, Cho ES, Petito CK. Price RW. Herpes zoster myelitis. Brain 1991: 1 14:1181-96.

8. Kalayjian RC, Cohen ML. Bonomo RA, Flanigan TP. Cytomegalovirus ventriculoencephalitis in AIDS: a syndrome with distinct clinical and pathologic features. Medicine (Baltimore) 1993; 72:67-77.

9. Hwang YM, Lee BI, Chung JW, Ahn JH, Kim KW, Kim DI. A case of herpes zoster myelitis: positive magnetic resonance imaging finding. Eur Neurol 1991:31:164-7.

10. Puchhammer-Stockl E. Popow-Kraupp T. Heinz FX. Mandl CW, Kunz C. Detection of varicella-zoster virus DNA by polymerase chain reaction in the cerebrospinal fluid of patients suffering from neurological complications associated with chicken pox or herpes zoster. J Clin Microbiol 1991;29:1513-6.

11. Shoji H, Honda Y, Murai I, Sato Y, Oizumi K, Hondo R. Detection of varicella-zoster virus DNA by polymerase chain reaction in cerebrospinal fluid of patients with herpes zoster meningitis. J Neurol 1992:239:69-70. 